\title{
Are all centers able to offer an adequate carcinological treatment for gastric carcinoma?
}

Keywords: Gastric carcinoma, multidisciplinary management, supportive care.

Abbreviations: FLOT, 5 fluoro-uracil, leucovorin, oxaliplatin, docetaxel; ECF, epirubicin, cisplatin, 5 fluoro-uracil; ECX, epirubicin, cisplatin, capecitabine; PF, cisplatin, 5 fluoro-uracil

\section{Opinion article}

Gastric carcinoma (GC) is the fourth most common malignancy worldwide and remains the second cause of death of all malignancies. Gastric cancer results from a combination of environmental factors along with specific genetic alterations. Multimodal approach from the diagnosis until the treatment (chemotherapy, radiotherapy and surgery) is necessary for an optimal outcome along with the implementation of novel promising agents.

Dysphagia, hematemesis, pain, anorexia, and dyspepsia are among the most common presenting symptoms of gastric cancer. Thus, the patients are initially referred to the gastroenterologist for further evaluation. The diagnosis of GC is made via upper gastro-intestinal endoscopy and biopsies. The pathologist must evaluate the specimen with microscopic morphologic and immune-histochemical studies. As all the cancers are now re-classified in different molecular genomic sub-types, as described in the cancer genome atlas (TCGA), the pathologic reports are becoming more challenging. However, outside a clinical trial, the recommendations are to test for human epidermal growth factor receptor 2 (Her-2) and program death ligand 1 (PDL1) expressions. ${ }^{1,2}$

Once the diagnosis is made, the next step is to do an optimal staging work up to differentiate between a localized $\mathrm{v} / \mathrm{s}$ a metastatic disease. Knowing that gastric carcinoma metastasizes most commonly to regional lymph nodes, peritoneum and liver, it is mandatory to exclude an advanced disease which will determine the later management and treatment plans: palliative non curative intent instead of aggressive multimodal curative approach. So, it is important to do a good echoendoscopy as well as a thorough evaluation for a distant dissemination: chest-abdomen-pelvis CT scan, PET CT scan, and in many institutions exploratory laparoscopy to exclude peritoneal carcinomatosis. Once the staging work up is done, every case must be discussed in a tumor board with the multidisciplinary contribution of the surgeon, medical oncologist, radiologist, radiotherapist, psychologist, dietitian and supportive care team. ${ }^{3,4}$

When the disease is localized and the objective of the treatment is curative, the patients must receive a perioperative chemotherapy followed by surgery, then a post-operative cytotoxic treatment (this strategy applies to all tumors >cT1 stage) Knowing that the current perioperative protocols are more aggressive than the previously used regimen (FLOT compared with ECF/ECX or FP), an adequate supportive management is mandatory in order to overcome the toxicities..$^{5-7}$ Surgeons and medical oncologists must keep in mind that this category of patients is usually in the 60s of age and many of them are smokers with other associated comorbidities. Moreover, they suffer from anorexia and malnutrition, either due to the malignancy related cachexia or to the obstructive effect of the tumor.

\author{
Volume 6 Issue 4 - 2018 \\ Maroun Sadek, Youssef Jounblat, Georges El \\ Hachem \\ Department of Medical Oncology, Institut Jules Bordet, Belgium
}

Correspondence: Georges El Hachem, MD. Hematologist and Medical Oncologist, Institut Jules Bordet, Brussels, Belgium, Email george.el.hashem@hotmail.com, georges.elhachem@bordet.be

Received: July 24, 2018| Published: August 17, 2018

Consequently, they must have a nutritionist consultation and close follow up to adapt their caloric intakes and avoid further weight loss that may complicate the treatment tolerance. The patients must benefit from a sufficient supportive care while receiving the chemotherapy: growth factors, antibiotics in case of febrile neutropenia, psychosocial help and management of other treatment related toxicities. Before proceeding to the surgical treatment, a re-staging work up is mandatory as many cases are micro-metastatic despite being staged as localized diseases, and surgeons must exclude non-operable and non-resectable cases. An incomplete surgery is more deleterious than leaving the non-resectable tumor while offering a non-surgical systemic strategy. The surgeons must have good skills in undergoing radical carcinologic surgeries in experienced centers: surgical techniques, negative margins, adequate lymph nodes dissection (N2 level) and management of the perioperative period which is crucial in determining post-operative complications, disease recurrence as well as overall survival. The surgical treatment is mutilating by itself, and the patients require a good post-surgical care: reanimation, nutritional support, rehabilitation, physical and psychosocial therapies. Unfortunately, $40 \%$ of the patients become frail, and won't be able to continue the post-operative chemotherapy. In this perspective, the supportive care team acts to help them overcoming the morbidities and toxicities of the surgery to let them achieve the pre-planned treatment. ${ }^{8,9}$ On the other side, many centers in the US adopt the perioperative chemo-radiation therapy and other centers in Asia use the modality of adjuvant chemotherapy treatment. Regardless of the therapeutic strategy, a multidisciplinary team discussion and decision are mandatory all over the treatment and disease courses. ${ }^{10,11}$

In the metastatic settings or when the patients are not candidates for surgery, the goal of the treatment will consist of a palliative chemotherapy and a preservation of the quality of life: psycho-social care, pain management, nutritional support, management of tumor related bleeding or stenosis. So, these patients must be addressed to the palliative/supportive care team as early as possible, because the early referral is correlated with a better outcome.

To conclude, gastric cancer remains a challenging disease with high potential of developing micro-metastases and generalized disease. Despite all the progresses, the 5-year survival doesn't exceed $20-25 \%$. 
An adequately performed, radical gastrectomy is the cornerstone of the treatment. Knowing the patients' comorbidities, the treatment related toxicities and the risks associated with the surgery, GC must be referred to experienced centers (Figure 1). The management of these tumors requires many surgical, medical and supportive skills, and so, is any center allowed to treat gastric cancer?

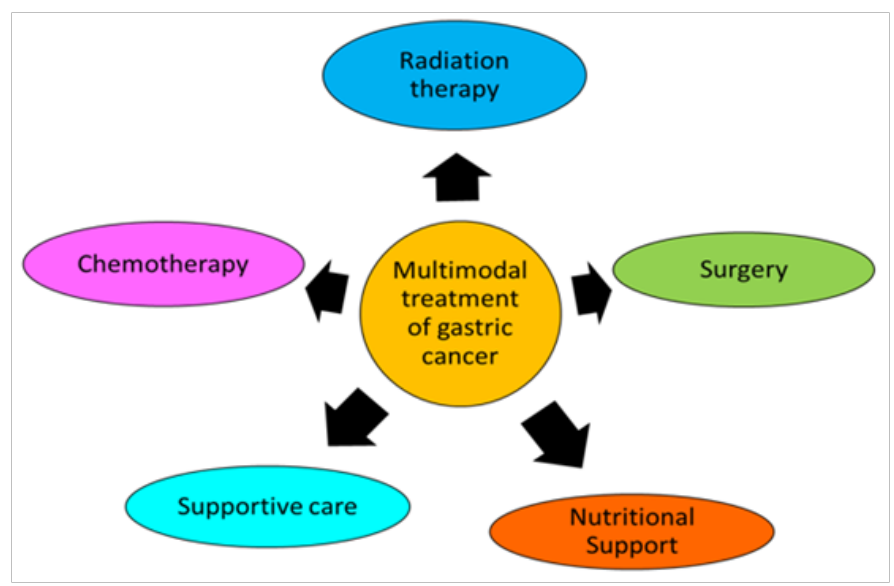

Figure I Multimodal multidisciplinary team management required in every tumor and specifically in gastric cancer for a better outcome and improved survival.

\section{Acknowledgements}

None.

\section{Conflict of interest}

All authors declare no conflicts of interest.

\section{References}

1. Jemal A, Bray F, Center MM, et al. Global cancer statistics. CA Cancer J Clin. 2011;61(2):69-90.

2. Arnold M, Karim-Kos HE, Coebergh JW, et al. Recent trends in incidence of Five common cancers in 26 European countries since 1988: analysis of the European Cancer Observatory. Eur J Cancer. 2015;51(9):1164-1187.
3. Boniface M, Wani S, Schefter T, et al. Multidisciplinary management for esophageal and gastric cancer. Cancer Manag Res. 2016;8:39-44.

4. Di L, Wu H, Zhu R, et al. Multi-disciplinary team for early gastric cancer diagnosis improves the detection rate of early gastric cancer. $B M C$ Gastroenterology. 2017;17(1):147.

5. Ychou M, Pignon JP, Lasser P. Phase III preliminary results of preoperative fluorouracil $(\mathrm{F})$ and cisplatin $(\mathrm{P})$ versus surgery alone in adenocarcinoma of stomach and lower esophagus (ASLE): FNLCC 94012-FFCD 9703 trial. Journal of Clinical Oncology. 2006;24(18 Suppl):S4026-S4026.

6. Cunningham D, Allum W, Stenning S, et al. Perioperative Chemotherapy versus Surgery Alone for Resectable Gastroesophageal Cancer. $N$ Engl J Med. 2006;355:11-20.

7. Al-Batran S, Homann N, SchmalenbergH, et al. Perioperative chemotherapy with docetaxel, oxaliplatin, and fluorouracil/leucovorin (FLOT) versus epirubicin, cisplatin, and fluorouracil or capecitabine (ECF/ECX) for resectable gastric or gastroesophageal junction (GEJ) adenocarcinoma (FLOT4-AIO): A multicenter, randomized phase 3 trial. Journal of Clinical Oncology. 2017;35(15 Suppl):4004.

8. Bozzetti F, Marubini E, Bonfanti G, et al. Total versus subtotal gastrectomy: surgical morbidity and mortality rates in a multicenter Italian randomized trial. The Italian Gastrointestinal Tumor Study Group. Ann Surg. 1997;226(5):613-620.

9. Da Costa WL, Coimbra FJ, Ribeiro HS, et al. Total gastrectomy for gastric cancer: an analysis of postoperative and long-term outcomes through time: results of 413 consecutive cases in a single cancer center. Annals of Surgical Oncology. 2014;22(3):750-757.

10. Macdonald JS, Smalley SR, Benedetti J, et al. Chemoradiotherapy after surgery compared with surgery alone for adenocarcinoma of the stomach or Gastroesophageal junction. N Engl J Med. 2001;345:725-730.

11. Bang YJ, Kim YW, Yang HK, et al. Adjuvant capecitabine and oxaliplatin for gastric cancer after D2 gastrectomy (CLASSIC): a phase 3 openlabel, randomised controlled trial. Lancet. 2012;379(9813):315-321. 